Kragujevac Journal of Mathematics

Volume 45(3) (2021), Pages 465-476.

\title{
QUANTITATIVE UNCERTAINTY PRINCIPLE FOR STURM-LIOUVILLE TRANSFORM
}

\author{
AHMED ABOUELAZ ${ }^{1}$, AZZEDINE ACHAK ${ }^{1 *}$, RADOUAN DAHER ${ }^{1}$, \\ AND NAJAT SAFOUANE ${ }^{1}$
}

\begin{abstract}
In this paper we consider the Sturm-Liouville transform $\mathcal{F}(f)$ on $\mathbb{R}_{+}$. We analyze the concentration of this transform on sets of finite measure. In particular, Donoho-Stark and Benedicks-type uncertainty principles are given.
\end{abstract}

\section{INTRODUCTION}

The uncertainty principle says that a function and its transform cannot concentrate both on small sets. Depending on the precise way to measure "concentration" and "smallness" this principle can assume different forms. This paper focuses on studying different uncertainty principles for the Sturm-Liouville transform, by following the procedures for similar transforms, such as the Fourier transform (the classical setting) we refer to the book [10] and the surveys [4,7] for further references. The concept of concentration has taken different interpretations in different contexts. For example: Benedicks [2], Slepian and Pollak [18], Landau and Pollak [13], and Donoho and Stark [6] paid attention to the supports of functions and gave quantitative uncertainty principles for the Fourier transforms. Qualitative uncertainty principles are not inequalities, but are theorems that tell us how a function (and its Fourier transform) behave under certain circumstances. For example: Hardy [11], Cowling and Price [5], Beurling [3], Miyachi [15] theorems enter within the framework of the quantitative uncertainty principles. The quantitative and qualitative uncertainty principles have been studied by many authors for various Fourier transforms, for examples (cf. $[1,11,14,16]$ ).

Key words and phrases. Sturm-Liouville transform, Benedicks theorem, Donoho-Stark's uncertainty principle.

2010 Mathematics Subject Classification. Primary: 42A38. Secondary: 44A35, 34B30.

DOI 10.46793/KgJMat2103.465A

Received: January 06, 2019.

Accepted: February 19, 2019. 
Our aim here is to consider uncertainty principles in which concentration is measured in sense of smallness of the support and when the transform under consideration is the Sturm-Liouville transform.

The first principle that is studied is a Donoho-Stark-type inequality. One can write the classical uncertainty principle in the following way: If a function $f(t)$ is essentially zero outside an interval of length $\Delta t$ and its Fourier transform $f(w)$ is essentially zero outside an interval of length $\Delta w$, then $\Delta t \Delta w \geq 1$. In [6], Donoho and Stark show that it is not necessary to assume that the support and the spectrum are concentrated on intervals and one can replace intervals by measurable sets, and then the length of the interval is naturally replaced by the measure of the set. In Section 2, a version of this inequality for the Sturm-Liouville transform is given, and, as it appears in [6] it is explained how to reconstruct a signal f from a noisy measurement, knowing that the signal is supported on a set $S$.

The second principle, studied in Section 3, is a Benedicks-type result which shows that two measurable sets $(S, \Sigma)$ with finite measure form a strong annihilating pair. This means that a function supported in $\mathrm{S}$ cannot have an spectrum in $\Sigma$ giving a quantitative information of the mass of a function whose spectrum is contained in $\Sigma$. The approach is based on the corresponding version of this type of principle for the integral operators transform, studied in [8]. A version of Benedicks type-inequality for integral operators transform with bounded and homogeneous kernel has been proved in [8]. In this paper, we consider a transform of a different nature where in particular the kernel is not homogeneous.

We recall that, Soltani in [19] study what is the relation between the measure and the spectrum of a function $f$ that is $\varepsilon$-concentrated in measurable sets giving. Concentration in support means that the part of the function that is not supported on a set is at least an $\varepsilon$ part of the total mass. The analogous version for spectrum states that the part of the spectrum not supported on a set is an $\varepsilon$ part of the total spectrum. It is shown that if a function is $\varepsilon$-concentrated in space and frequency, then the product of the measures of the support and spectrum is lower bounded by a number close to one.

In order to describe our results, we first need to introduce some facts about harmonic analysis related to Sturm-Liouville transform. We cite here, as briefly as possible, some properties. For more details we refer to [19].

The Sturm-Liouville operator $\Delta$ defined on $\mathbb{R}_{+}$by

$$
\Delta=\frac{\partial^{2}}{\partial x^{2}}+\frac{\mathcal{A}^{\prime}(x)}{\mathcal{A}(x)} \frac{\partial}{\partial x}+\rho^{2}
$$

where $\rho$ is a nonnegative real number and $\mathcal{A}(x)=x^{2 \alpha+1} B(x), \alpha>-\frac{1}{2}$, where $B$ is a positive, even, infinitely differentiable function on $\mathbb{R}$ such that $B(0)=1$. Moreover, we assume that $\mathcal{A}$ and $B$ satisfy the following conditions:

- $\mathcal{A}$ is increasing and $\lim _{x \rightarrow \infty} \mathcal{A}(x)=\infty$; 
- $\frac{\mathcal{A}^{\prime}}{\mathcal{A}}$ is decreasing and $\lim _{x \rightarrow \infty} \frac{\mathcal{A}^{\prime}(x)}{\mathcal{A}(x)}=2 \rho$;

- there exists a constant $\delta>0$ such that

$$
\frac{\mathcal{A}^{\prime}(x)}{\mathcal{A}(x)}= \begin{cases}2 \rho+D(x) \exp (-\delta x), & \text { if } \rho>0, \\ \frac{2 \alpha+1}{x}+D(x) \exp (-\delta x), & \text { if } \rho=0,\end{cases}
$$

where $D$ is an infinitely differentiable function on $] 0, \infty[$, bounded and with bounded derivatives on all intervals $\left[x_{0}, \infty\left[\right.\right.$ for $x_{0}>0$. For all $\lambda \in \mathbb{C}$ the equation

$$
\left\{\begin{array}{l}
\Delta u=-\lambda^{2} u \\
u(0)=1, \quad u^{\prime}(0)=0,
\end{array}\right.
$$

admits a unique solution denoted $\varphi_{\lambda}$, with the following properties:

- for $x \geq 0$ the function $\lambda \mapsto \varphi_{\lambda}(x)$ is analytic on $\mathbb{C}$;

- for $\lambda \in \mathbb{C}$ the function $\lambda \mapsto \varphi_{\lambda}(x)$ is even and infinitely differentiable on $\mathbb{R}$;

- $\left|\varphi_{\lambda}(x)\right| \leq 1$ for all $\lambda, x \in \mathbb{R}$.

For nonzero $\lambda \in \mathbb{C}$ the equation $\Delta u=-\lambda^{2} u$ has a solution $\Phi_{\lambda}$ satisfying

$$
\Phi_{\lambda}(x)=\frac{1}{\sqrt{\mathcal{A}(x)}} \exp (i \lambda x) V(x, \lambda),
$$

with $\lim _{x \rightarrow \infty} V(x, \lambda)=1$. Consequently, there exists a function $\lambda \mapsto c(\lambda)$, such that

$$
\varphi_{\lambda}=c(\lambda) \Phi_{\lambda}+c(-\lambda) \Phi_{-\lambda}, \quad \text { for nonzero } \lambda \in \mathbb{C} .
$$

Moreover, there exist positive constants $k_{1}, k_{2}$ and $k$ such that

$$
k_{1}|\lambda|^{2 \alpha+1} \leq|c(\lambda)|^{-2} \leq k_{2}|\lambda|^{2 \alpha+1},
$$

for all $\lambda$ such that $\operatorname{Im} \lambda \leq 0$ and $|\lambda| \geq k$.

Let us introduce the dilation operator $D_{\rho}, \rho>0$, defined by

$$
D_{\rho} f(x)=\frac{1}{\rho^{\alpha+1}} f\left(\frac{x}{\rho}\right) \text {. }
$$

We denote by $L^{p}\left(\mathbb{R}_{+}, \mu\right), 1 \leq p \leq \infty$, the space of measurable functions $f$ on $\mathbb{R}_{+}$ such that

$$
\begin{aligned}
\|f\|_{L^{p}\left(\mathbb{R}_{+}, \mu\right)} & =\left(\int_{\mathbb{R}_{+}}|f(x)|^{p} d \mu(x)\right)^{\frac{1}{p}}<+\infty, \quad \text { if } 1 \leq p<+\infty \\
\|f\|_{\infty} & =\operatorname{esssup}_{x \in \mathbb{R}_{+}}|f(x)|<+\infty, \quad \text { if } p=\infty
\end{aligned}
$$

where $d \mu(x)=\mathcal{A}(x) d x$.

The Sturm-Liouville transform $\mathcal{F}$ is defined on $L^{1}\left(\mathbb{R}_{+}, \mu\right)$ by

$$
\mathcal{F}(f)(\lambda)=\int_{\mathbb{R}_{+}} f(x) \varphi_{\lambda}(x) d \mu(x), \quad \text { for all } \lambda \in \mathbb{R} .
$$

Let $\nu$ the measure defined on $\left[0, \infty\left[\right.\right.$ by $d \nu(\lambda)=\frac{d \lambda}{2 \pi|c(\lambda)|^{2}}$ and by $L^{p}(\nu), 1 \leq p \leq \infty$, the space of measurable functions $f$ on $\left[0, \infty\left[\right.\right.$, such that $\|f\|_{L^{p}\left(\mathbb{R}_{+}, \nu\right)}<\infty$. 
For all $f \in L^{1}\left(\mathbb{R}_{+}, \mu\right)$, the function $\mathcal{F}(f)$ is continuous on $\mathbb{R}$ and we have

$$
\|\mathcal{F}(f)\|_{L^{\infty}\left(\mathbb{R}_{+}, \nu\right)} \leq\|f\|_{L^{1}\left(\mathbb{R}_{+}, \mu\right)} .
$$

Theorem 1.1 (Plancherel theorem). The Sturm-Liouville transform $\mathcal{F}$ extends uniquely to an isometric isomorphism of $L^{2}\left(\mathbb{R}_{+}, \mu\right)$ onto $L^{2}\left(\mathbb{R}_{+}, \nu\right)$

$$
\int_{\mathbb{R}_{+}}|f(x)|^{2} d \mu(x)=\int_{\mathbb{R}_{+}}|\mathcal{F}(f)(\lambda)|^{2} d \nu(\lambda) .
$$

Theorem 1.2 (Inversion theorem). Let $f \in L^{1}\left(\mathbb{R}_{+}, \mu\right)$ such that $\mathcal{F}(f) \in L^{1}\left(\mathbb{R}_{+}, \nu\right)$. Then

$$
f(x)=\int_{\mathbb{R}_{+}} \mathcal{F}(f)(\lambda) \varphi_{\lambda}(x) d \nu(\lambda) \text { a.e. } x \in \mathbb{R}^{+} .
$$

Theorem 1.3 (Riesz's interpolation theorem). Let $f \in L^{p}\left(\mathbb{R}_{+}>\mu\right)$. Then we get the Hausdorff-Young inequality (see [20]) $\|\mathcal{F}(f)\|_{L^{q}\left(\mathbb{R}_{+}, \nu\right)} \leq\|f\|_{L^{p}\left(\mathbb{R}_{+}, \mu\right)}$.

Definition 1.1. Let $S, \Sigma$ be two measurable subsets of $\mathbb{R}_{+}^{d}$. Then $(S, \Sigma)$ is called a weak annihilating pair for the Sturm-Liouville transform if $\operatorname{supp} f \subset S$ and $\operatorname{supp} \mathcal{F}_{k, \alpha}(f) \subset \Sigma$, implies that $f=0$, where $\operatorname{supp} f=\{x: f(x) \neq 0\}$.

Definition 1.2. Let $S, \Sigma$ be two measurable subsets of $\mathbb{R}_{+}$. Then $(S, \Sigma)$ is called a strong annihilating pair for the Sturm-Liouville transform if there exists a constant $C(S, \Sigma)$ such that for all function $f \in L^{2}\left(\mathbb{R}_{+}, \mu\right)$, with $\operatorname{supp} \mathcal{F}(f) \subset \Sigma$,

$$
\|f\|_{L^{2}\left(\mathbb{R}_{+}, \mu\right)} \leq C(S, \Sigma)\|f\|_{L^{2}\left(S^{c}, \mu\right)},
$$

where $S^{c}=\mathbb{R}_{+} \backslash S$ and $\operatorname{supp} f=\{x: f(x) \neq 0\}$.

\section{The Donoho-Strak's Uncertainty Principle}

The classical uncertainty principle says that if a function $f(t)$ is essentially zero outside an interval of light $\Delta t$ and its Fourier transform $\hat{f}(w)$ is essentially zero outside an interval of length $\Delta w$, then $\Delta t \Delta w \geq 1$. In this section we will prove a quantitative uncertainty inequality about the essential supports of a nonzero function $f \in L^{2}\left(\mathbb{R}_{+}, \mu\right)$ and its Sturm-Liouville transform.

The first such inequality for the usual Fourier transform was obtained by DonohoStark [6].

We consider a pair of orthogonal projections on $L^{2}\left(\mathbb{R}_{+}, \mu\right)$ defined by $P_{S} f=\chi_{S} f$, $Q_{\Sigma} f=\mathcal{F}^{-1}\left[\chi_{\Sigma} \mathcal{F}(f)\right]$, where $S$ and $\Sigma$ are measurable subsets of $\mathbb{R}_{+}$, and $\chi_{S}$ denote the characteristic function of $S$.

Let $0<\varepsilon_{S}, \varepsilon_{\Sigma}<1$ and let $f \in L^{2}\left(\mathbb{R}_{+}, \mu\right)$ be a nonzero function. We say that $f$ is $\varepsilon_{S^{-}}$-time-limited on $S$ if $\left\|P_{S^{c}} f\right\|_{L^{2}\left(\mathbb{R}_{+}, \mu\right)} \leq \varepsilon_{S}\|f\|_{L^{2}\left(\mathbb{R}_{+}, \mu\right)}$. Similarly, we say that $f$ is $\varepsilon_{\Sigma^{-}}$-band-limited on $\Sigma$ for the Sturm-Liouville transform if $\left\|Q_{\Sigma^{c}} f\right\|_{L^{2}\left(\mathbb{R}_{+}, \mu\right)} \leq$ $\varepsilon_{\Sigma}\|f\|_{L^{2}\left(\mathbb{R}_{+}, \mu\right)}$.

We denote by $P_{S} \cap Q_{\Sigma}$ for the orthogonal projection onto the intersection of the ranges of $P_{S}$ and $Q_{\Sigma}$, we will write $\operatorname{Im} T$ for the range of a linear operator $T$. We 
denote by $\|T\|_{H S}$ the Hilbert-Schmidt norm of the linear operator $T$. The definition of this norm [21, page 262] implies that for any pair of projections $E, F$ one has

$$
\operatorname{dim}\left(\operatorname{Im} P_{S} \cap \operatorname{Im} Q_{\Sigma}\right)=\left\|P_{S} \cap Q_{\Sigma}\right\|_{H S}^{2} \leq\left\|P_{S} Q_{\Sigma}\right\|_{H S}^{2}
$$

Theorem 2.1. Let $\Sigma, S \subset \mathbb{R}_{+}$be a pair of measurable subsets and let $\varepsilon_{S}, \varepsilon_{\Sigma}>0$ such that $\varepsilon_{S}^{2}+\varepsilon_{\Sigma}^{2}<1$. Let $f \in L^{2}\left(\mathbb{R}_{+}, \mu\right)$ be a non function. If $f$ is $\varepsilon_{S}$-time-limited on $S$ and $\varepsilon_{\Sigma}$-band-limited on $\Sigma$ for the Sturm-Liouville transform, then

$$
\mu(S) \nu(\Sigma) \geq\left(1-\sqrt{\varepsilon_{S}^{2}+\varepsilon_{\Sigma}^{2}}\right)^{2} .
$$

We will need the following well-known lemma.

Lemma 2.1. Let $(S, \Sigma)$ be two measurable subsets of $\mathbb{R}_{+}$. Then the following assertions are equivalent.

i) $\left\|P_{S} Q_{\Sigma}\right\|=\left\|P_{S} Q_{\Sigma}\right\|_{L^{2}\left(\mathbb{R}_{+}, \mu\right)}<1$.

ii) $(S, \Sigma)$ is strongly annihilating pair for the Sturm-Liouville transform. Moreover, we have $\|f\|_{L^{2}\left(\mathbb{R}_{+}, \mu\right)}^{2} \leq\left(1-\left\|P_{S} Q_{\Sigma}\right\|\right)^{-2}\left(\left\|P_{S^{c}} f\right\|_{L^{2}\left(\mathbb{R}_{+}, \mu\right)}^{2}+\left\|Q_{\Sigma^{c}} f\right\|_{L^{2}\left(\mathbb{R}_{+}, \mu\right)}^{2}\right)$.

Proof. Firstly we show the following implication i) $\Rightarrow$ ii). The identity operator $I$ satisfies $I=P_{S}+P_{S^{c}}=P_{S} Q_{\Sigma}+P_{S} Q_{\Sigma^{c}}+P_{S^{c}}$, we have from the orthogonality of $P_{S}$ and $P_{S^{c}}$

$$
\begin{aligned}
\left\|f-P_{S} Q_{\Sigma} f\right\|_{L^{2}\left(\mathbb{R}_{+}, \mu\right)}^{2} & =\left\|P_{S} Q_{\Sigma^{c}} f+P_{S^{c}} f\right\|_{L^{2}\left(\mathbb{R}_{+}, \mu\right)}^{2} \\
& =\left\|P_{S} Q_{\Sigma^{c}} f\right\|_{L^{2}\left(\mathbb{R}_{+}, \mu\right)}^{2}+\left\|P_{S^{c}} f\right\|_{L^{2}\left(\mathbb{R}_{+}, \mu\right)}^{2} .
\end{aligned}
$$

It follows, by $\left\|P_{S}\right\|=1$, that

$$
\left\|f-P_{S} Q_{\Sigma} f\right\|_{L^{2}\left(\mathbb{R}_{+}, \mu\right)} \leq\left(\left\|Q_{\Sigma^{c}} f\right\|_{L^{2}\left(\mathbb{R}_{+}, \mu\right)}^{2}+\left\|P_{S^{c}} f\right\|_{L^{2}\left(\mathbb{R}_{+}, \mu\right)}^{2}\right)^{\frac{1}{2}} .
$$

On the other hand, we have

$$
\begin{aligned}
\left\|f-P_{S} Q_{\Sigma} f\right\|_{L^{2}\left(\mathbb{R}_{+}, \mu\right)} & \geq\|f\|_{L^{2}\left(\mathbb{R}_{+}, \mu\right)}-\left\|P_{S} Q_{\Sigma} f\right\|_{L^{2}\left(\mathbb{R}_{+}, \mu\right)} \\
& \geq\|f\|_{L^{2}\left(\mathbb{R}_{+}, \mu\right)}-\left\|P_{S} Q_{\Sigma}\right\|\|f\|_{L^{2}\left(\mathbb{R}_{+}, \mu\right)} .
\end{aligned}
$$

It follows, from inequality $(2.2)$,

$$
\left(1-\left\|P_{S} Q_{\Sigma}\right\|\right)\|f\|_{L^{2}\left(\mathbb{R}_{+}, \mu\right)} \leq\left(\left\|P_{S^{c}} f\right\|_{L^{2}\left(\mathbb{R}_{+}, \mu\right)}^{2}+\left\|Q_{\Sigma^{c}} f\right\|_{L^{2}\left(\mathbb{R}_{+}, \mu\right)}^{2}\right)^{\frac{1}{2}}
$$

As $\left\|P_{S} Q_{\Sigma}\right\|<1$, then we obtain the desired result. 
Let us now show the second implication ii) $\Rightarrow$ i). Recall that

$$
\begin{aligned}
\left\|P_{S} Q_{\Sigma}\right\|=\left\|Q_{\Sigma} P_{S}\right\| & =\sup _{f \in L^{2}\left(\mathbb{R}_{+}, \mu\right)} \frac{\left\|Q_{\Sigma} P_{S} f\right\|_{L^{2}\left(\mathbb{R}_{+}, \mu\right)}}{\|f\|_{L^{2}\left(\mathbb{R}_{+}, \mu\right)}} \\
& =\sup _{f: f=P_{S} f} \frac{\left\|Q_{\Sigma} f\right\|_{L^{2}\left(\mathbb{R}_{+}, \mu\right)}}{\|f\|_{L^{2}\left(\mathbb{R}_{+}, \mu\right)}} \\
& =\sup _{f: f=Q_{\Sigma} f} \frac{\left\|P_{S} f\right\|_{L^{2}\left(\mathbb{R}_{+}, \mu\right)}}{\|f\|_{L^{2}\left(\mathbb{R}_{+}, \mu\right)}} \\
& <1 .
\end{aligned}
$$

We suppose that $\left\|P_{S} Q_{\Sigma}\right\|=1$. Then we can find a bandlimited sequence $f_{n} \in$ $L^{2}\left(\mathbb{R}_{+}, \mu\right)$ on $\Sigma$ of norm 1 (in particular $f_{n}=Q_{\Sigma} f_{n}$ ) such that

$$
\left\|P_{S} f_{n}\right\|_{L^{2}\left(\mathbb{R}_{+}, \mu\right)} \rightarrow 1 \text { as } n \rightarrow \infty \text {. }
$$

By the orthogonality of $S$, we have

$$
\left\|P_{S^{c}} f_{n}\right\|_{L^{2}\left(\mathbb{R}_{+}, \mu\right)}^{2}=\left\|f_{n}\right\|_{L^{2}\left(\mathbb{R}_{+}, \mu\right)}^{2}-\left\|P_{S} f_{n}\right\|_{L^{2}\left(\mathbb{R}_{+}, \mu\right)} \rightarrow 0 \text { as } n \rightarrow \infty,
$$

which contradicts (1.3).

Lemma 2.2. If $0<\mu(S) \nu(\Sigma)<1$, then for all function $f \in L^{2}\left(\mathbb{R}_{+}, \mu\right)$ such that $\operatorname{supp} \mathcal{F}(f) \subset \Sigma$ we have

$$
\|f\|_{L^{2}\left(\mathbb{R}_{+}, \mu\right)} \leq(1-\sqrt{\mu(S) \nu(\Sigma)})^{-1}\|f\|_{L^{2}\left(S^{c}, \mu\right)} .
$$

Proof. A straightforward computation shows that $P_{S} Q_{\Sigma}$ is an integral operator with kernel $N(t, x)=\chi_{S}(t) \mathcal{F}^{-1}\left(\chi_{\Sigma} \varphi_{\lambda}(t)\right)(x)$. Indeed, we have

$$
\begin{aligned}
P_{S} Q_{\Sigma} f(t) & =\chi_{S}(t) \int_{\mathbb{R}_{+}} \chi_{\Sigma}(\xi) \mathcal{F}(f)(\xi) \varphi_{\lambda}(t) d \nu(\xi) \\
& =\chi_{S}(t) \int_{\mathbb{R}_{+}} \chi_{\Sigma}(\xi) \varphi_{\lambda}(t)\left(\int_{\mathbb{R}_{+}} f(x) \varphi_{\lambda}(x) d \mu(x)\right) d \nu(\xi) \\
& =\int_{\mathbb{R}_{+}} f(x) N(t, x) d \mu(x),
\end{aligned}
$$

where

$$
N(t, x)=\chi_{S}(t) \int_{\mathbb{R}_{+}} \chi_{\Sigma}(\xi) \varphi_{\lambda}(t) \varphi_{\lambda}(x) d \nu(\xi) .
$$

Since, $\nu(\Sigma)<\infty$ and $\varphi_{\lambda}$ is bounded, then for all $t \in \mathbb{R}_{+}, \chi_{\Sigma} \varphi_{\lambda}(t) \in L^{2}\left(\mathbb{R}_{+}, \nu\right)$. Then $P_{S} Q_{\Sigma}$ is an integral operator with kernel $N(t, x)=\chi_{S}(t) \mathcal{F}^{-1}\left(\chi_{\Sigma} \varphi_{\lambda}(t)\right)(x)$. As $\left\|P_{S} Q_{\Sigma}\right\|_{H S}=\|N\|_{L^{2}\left(\mathbb{R}_{+} \times \mathbb{R}_{+}, \mu \otimes \mu\right)}$, it follows from Plancherel's theorem 1.2 that

$$
\begin{aligned}
\left\|P_{S} Q_{\Sigma}\right\|_{H S}^{2} & =\int_{\mathbb{R}_{+}}\left|\chi_{S}(t)\right|^{2}\left(\int_{\mathbb{R}_{+}}\left|\mathcal{F}^{-1}\left(\chi_{\Sigma} \varphi_{\lambda}(t)\right)(x)\right|^{2} d \mu(\xi)\right) d \mu(t) \\
& =\int_{\mathbb{R}_{+}} \chi_{S}(t) \int_{\mathbb{R}_{+}} \chi_{\Sigma}(\xi)\left|\varphi_{\lambda}(t)\right|^{2} d \nu(\xi) d \mu(t) .
\end{aligned}
$$


We can deduce from $\left|\varphi_{\lambda}(t)\right|<1$ that

$$
\left\|P_{S} Q_{\Sigma}\right\| \leq\left\|P_{S} Q_{\Sigma}\right\|_{H S} \leq \sqrt{\mu(S) \nu(\Sigma)}
$$

Since $\mu(S) \nu(\Sigma)<1$, then we have from inequality (2.4) and Lemma 2.1

$$
\|f\|_{L^{2}\left(\mathbb{R}_{+}, \mu\right)}^{2} \leq(1-\sqrt{\mu(S) \nu(\Sigma)})^{-2}\left(\left\|P_{S^{c}} f\right\|_{L^{2}\left(\mathbb{R}_{+}, \mu\right)}^{2}+\left\|Q_{\Sigma^{c}} f\right\|_{L^{2}\left(\mathbb{R}_{+}, \mu\right)}^{2}\right) .
$$

Since supp $\mathcal{F}(f) \subset \Sigma$, it follows from Plancherel's theorem 1.2 that

$$
\left\|Q_{\Sigma^{c}} f\right\|_{L^{2}\left(\mathbb{R}_{+}, \mu\right)}^{2}=\int_{\Sigma^{c}}|\mathcal{F}(\xi)|^{2} d \nu(\xi)=\|\mathcal{F}(f)\|_{L^{2}\left(\Sigma^{c}, \nu\right)}^{2}=0
$$

which shows the desired result.

Proof of Theorem 2.1. The result follows from inequalities (2.3) and (2.4). Indeed, $f$ is $\varepsilon_{S^{-}}$-time-limited on $S$, then $\left\|P_{S^{c}} f\right\|_{L^{2}\left(\mathbb{R}^{2}, \mu\right)} \leq \varepsilon_{S}\|f\|_{L^{2}\left(\mathbb{R}^{2}, \mu\right)} . f$ is $\varepsilon_{\Sigma^{-}}$-band-limited on $\Sigma$ for the Sturm-Liouville transform, then $\left\|Q_{\Sigma^{c}} f\right\|_{L^{2}\left(\mathbb{R}^{2}, \mu\right)} \leq \varepsilon_{\Sigma}\|f\|_{L^{2}\left(\mathbb{R}^{2}, \mu\right)}$. It follows that

$$
\left\|P_{S^{c}} f\right\|_{L^{2}\left(\mathbb{R}^{2}, \mu\right)}^{2}+\left\|Q_{\Sigma^{c}} f\right\|_{L^{2}\left(\mathbb{R}^{2}, \mu\right)}^{2} \leq\left(\varepsilon_{S}^{2}+\varepsilon_{\Sigma}^{2}\right)\|f\|_{L^{2}\left(\mathbb{R}^{2}, \mu\right)}^{2},
$$

from (2.3) we deduce that $\left(1-\left\|P_{S} Q_{\Sigma}\right\|\right)^{2} \leq \varepsilon_{S}^{2}+\varepsilon_{\Sigma}^{2}$. It follows, from (2.4), that $1-\sqrt{\varepsilon_{S}^{2}+\varepsilon_{\Sigma}^{2}} \leq\left\|P_{S} Q_{\Sigma}\right\| \leq \sqrt{|S||\Sigma|}$, which proves the desired result.

Remark 2.1. From inequalities (2.1) and (2.4) it follows that

$$
\operatorname{dim}\left(\operatorname{Im} P_{S} \cap \operatorname{Im} Q_{\Sigma}\right) \leq\left\|P_{S} Q_{\Sigma}\right\|_{H S}^{2}<\infty
$$

The following example is prototypical. A signal $f$ is transmitted to a receiver who know that $f$ is bandlimited on $S$ for the Sturm-Liouville transform, meaning that $f$ is synthesized using only frequency on $S$; equivalently $f=Q_{\Sigma} f$. Suppose that the observation of $f$ is corrupted by a noise $n \in L^{2}\left(\mathbb{R}_{+}, \mu\right)$ (which is nonetheless assumed to be small) and an unregistered values on $S$. Thus, the observable function $r$ satisfies

$$
r(x)= \begin{cases}f(x)+n(x), & x \in S^{c}, \\ 0, & x \in S .\end{cases}
$$

Here, we have assumed without loss of generality that $n=0$ on $S$. Equivalently, $r=\left(I-P_{S}\right) f+n$. We say that $f$ can be stably reconstructed from $r$, if there exists a linear operator $K$ and a constant $C$ such that

$$
\|f-K r\|_{L^{2}\left(\mathbb{R}_{+}, \mu\right)} \leq C\|n\|_{L^{2}\left(\mathbb{R}_{+}, \mu\right)} .
$$

The estimate (2.7) shows that the noise $n$ is at most amplified by a factor $C$.

Corollary 2.1. If $S$ and $\Sigma$ are arbitrary measurable sets of $\mathbb{R}_{+}$with $0<\mu(S) \nu(\Sigma)<$ 1 , then $f$ can be stably reconstructed from $r$. The constant $C$ in equation (2.7) is not larger than $(1-\sqrt{\mu(S) \nu(\Sigma)})^{-1}$. 
Proof. If $\mu(S) \nu(\Sigma)<1$, using (2.4), $\left\|P_{S} Q_{\Sigma}\right\|<1$. Hence, $I-P_{S} Q_{\Sigma}$ is invertible. Let $K=\left(I-P_{S} Q_{\Sigma}\right)^{-1}$. Since $f$ is bandlimited on $\Sigma$, then $\left(I-P_{S}\right) f=\left(I-P_{S} Q_{\Sigma}\right) f$. Therefore,

$$
\begin{aligned}
f-K r & =f-K\left(\left(I-P_{S}\right) f+n\right) \\
& =f-K\left(I-P_{S} Q_{\Sigma}\right) f-K n \\
& =f-\left(I-P_{S} Q_{\Sigma}\right)\left(I-P_{S} Q_{\Sigma}\right)^{-1} f-K n \\
& =0-K n .
\end{aligned}
$$

So, that

$$
\begin{aligned}
\|f-K r\|_{L^{2}\left(\mathbb{R}_{+}, \mu\right)} & =\|K n\|_{L^{2}\left(\mathbb{R}_{+}, \mu\right)} \\
& \leq\left\|\left(I-P_{S} Q_{\Sigma}\right)^{-1}\right\|\|n\|_{L^{2}\left(\mathbb{R}_{+}, \mu\right)} \\
& \leq \sum_{k=0}^{\infty}\left\|P_{S} Q_{\Sigma}\right\|^{k}\|n\|_{L^{2}\left(\mathbb{R}_{+}, \mu\right)} \\
& \leq \sum_{k=0}^{\infty}(\mu(S) \nu(\Sigma))^{\frac{k}{2}}\|n\|_{L^{2}\left(\mathbb{R}_{+}, \mu\right)} \\
& =(1-\sqrt{\mu(S) \nu(\Sigma)})^{-1}\|n\|_{L^{2}\left(\mathbb{R}_{+}, \mu\right)} .
\end{aligned}
$$

The constant $C$ in equation $(2.7)$ is therefore not larger than $\left(1-\sqrt{\mu(S) \nu(\Sigma)}^{-1}\right.$.

The identity $K=\left(I-P_{S} Q_{\Sigma}\right)^{-1}=\sum_{k=0}^{\infty}\left(P_{S} Q_{\Sigma}\right)^{k}$ suggests an algorithm for computing $K r$. Put $f^{(n)}=\sum_{k=0}^{n}\left(P_{S} Q_{\Sigma}\right)^{k} r$, then

$$
f^{(0)}=r, \quad f^{(n+1)}=r+P_{S} Q_{\Sigma} f^{(n)} \quad \text { and } \quad f^{(n)} \rightarrow K r \text { as } n \rightarrow \infty .
$$

As $f$ is bandlimited on $\Sigma$ we deduce that

$$
f^{(n+1)}-f=P_{S} Q_{\Sigma}\left(f^{(n)}-f\right) .
$$

Algorithms of this type have applied to a host of problems in signal recovery (see for examples $[12,17])$.

\section{UnCERTAinty PRINCIPLES}

In this section we will give some remarks about annihilating sets.

Proposition 3.1. Let $f \in L^{2}\left(\mathbb{R}_{+}, \mu\right)$ has non empty support, then

$$
\nu(\operatorname{supp} \mathcal{F}) \mu(\operatorname{supp} f) \geq 1 .
$$

In particular, if $\mu(\operatorname{supp} f) \nu(\operatorname{supp} \mathcal{F})<1$, then $f=0$. 
Proof. If the function $f \in L^{2}\left(\mathbb{R}_{+}, \mu\right)$ has non empty support, by the Cauchy-Schwartz inequality and (1.1), we have

$$
\begin{aligned}
\|\mathcal{F}\|_{L^{2}\left(\mathbb{R}_{+}, \nu\right)}^{2} & \leq \nu(\operatorname{supp} \mathcal{F}(f))\|\mathcal{F}(f)\|_{\infty}^{2} \\
& \leq \nu(\operatorname{supp} \mathcal{F}(f))\|f\|_{L^{1}\left(\mathbb{R}_{+}, \mu\right)}^{2} \\
& \leq \nu(\operatorname{supp} \mathcal{F}(f)) \mu(\operatorname{supp} f)\|f\|_{L^{2}\left(\mathbb{R}_{+}, \mu\right)}^{2} .
\end{aligned}
$$

Using Plancherel's theorem 1.2 we have the following quantitative uncertainty inequality connecting the support of $f$ and the support of its Sturm-Liouville transform $\mathcal{F}$

$$
\nu(\operatorname{supp} \mathcal{F}) \mu(\operatorname{supp} f) \geq 1 \text {. }
$$

It follows that if $\mu(\operatorname{supp} f) \nu(\operatorname{supp} \mathcal{F})<1$, then $f=0$.

Proposition 3.2. Let $f \in L^{1}\left(\mathbb{R}_{+}, \mu\right) \cap L^{p}\left(\mathbb{R}_{+}, \mu\right), 1<p \leq 2$, then

$$
\|\mathcal{F}\|_{L^{q}\left(\mathbb{R}_{+}, \nu\right)} \leq \nu(\operatorname{supp} \mathcal{F}(f))^{1 / q} \mu(\operatorname{supp} f)^{1 / q}\|f\|_{L^{p}\left(\mathbb{R}_{+}, \mu\right)},
$$

where $q=\frac{p}{p-1}$.

Proof. Let $f \in L^{1}\left(\mathbb{R}_{+}, \mu\right) \cap L^{p}\left(\mathbb{R}_{+}, \mu\right), 1<p \leq 2$, then by Hölder's inequality and (1.1), we get

$$
\begin{aligned}
\|\mathcal{F}(f)\|_{L^{q}\left(\mathbb{R}_{+}, \nu\right)} & \leq \nu(\operatorname{supp} \mathcal{F}(f))^{1 / q}\|\mathcal{F}(f)\|_{\infty} \\
& \leq \nu(\operatorname{supp} \mathcal{F}(f))^{1 / q}\|f\|_{L^{1}\left(\mathbb{R}_{+}, \mu\right)} \\
& \leq \nu(\operatorname{supp} \mathcal{F}(f))^{1 / q} \mu(\operatorname{supp} f)^{1 / q}\|f\|_{L^{p}\left(\mathbb{R}_{+}, \mu\right)}
\end{aligned}
$$

Proposition 3.3. Let $f \in L^{2}\left(\mathbb{R}_{+}, \mu\right) \cap L^{p}\left(\mathbb{R}_{+}, \mu\right), 1<p \leq 2$, then

$$
1<\nu(\operatorname{supp} \mathcal{F}(f))^{\frac{q-2}{2 q}} \mu(\operatorname{supp} f)^{\frac{2-p}{2 p}},
$$

where $q=\frac{p}{p-1}$.

Proof. Let $f \in L^{2}\left(\mathbb{R}_{+}, \mu\right) \cap L^{p}\left(\mathbb{R}_{+}, \mu\right), 1<p \leq 2$, then by (1.1), Hölder's inequality and Riez's interpolation, we get

$$
\begin{aligned}
\|\mathcal{F}(f)\|_{L^{2}\left(\mathbb{R}_{+}, \nu\right)} & \leq \nu(\operatorname{supp} \mathcal{F}(f))^{\frac{q-2}{2 q}}\|\mathcal{F}(f)\|_{L^{q}\left(\mathbb{R}_{+}, \nu\right)} \\
& \leq \nu(\operatorname{supp} \mathcal{F}(f))^{\frac{q-2}{2 q}}\|f\|_{L^{p}\left(\mathbb{R}_{+}, \mu\right)} \\
& \leq \nu(\operatorname{supp} \mathcal{F}(f))^{\frac{q-2}{2 q}} \mu(\operatorname{supp} f)^{\frac{2-p}{2 p}}\|f\|_{L^{2}\left(\mathbb{R}_{+}, \mu\right)},
\end{aligned}
$$

by Plancherel's formula we get the desired result.

Lemma 3.1. Any nonzero function in $\mathfrak{C}_{0}\left(\mathbb{R}_{+}^{d}\right)$ has linearly independent dilates.

Proof. In the case $d=1$ this Lemma was proved in [8]. The case $d>1$ we reduce to the case $d=1$. Let $f \in \mathcal{C}_{0}\left(\mathbb{R}_{+}^{d}\right)$ such that $f \neq 0$, if $x=\left(x_{1}, \ldots, x_{d-1}, 0\right)=r\left(x^{\prime}, 0\right) \in \mathbb{R}_{+}^{d}$, $\sum_{i=1}^{d-1}\left|x_{i}^{\prime}\right|^{2}=1, r \in \mathbb{R}_{+}$, we get $g(r)=f\left(r\left(x^{\prime}, 0\right)\right)$.

If $x_{d}>0, x=r\left(\theta x^{\prime}, 1\right)$, where $\sum_{i=1}^{d-1}\left|x_{i}^{\prime}\right|^{2}=1$, and $r, \theta \in \mathbb{R}^{+}$, we get $g(r)=$ $f\left(r\left(\theta x^{\prime}, 1\right)\right)$. In both cases $g(r) \in \mathcal{C}_{0}\left(\mathbb{R}_{+}\right)$. 
Lemma 3.2. Let $S_{0}$ and $\Sigma_{0}$ be a pair of measurable subsets of $\mathbb{R}_{+}$with $0<\mu\left(S_{0}\right), \nu\left(\Sigma_{0}\right)$ $<\infty$, then exist an infinite sequence of distinct numbers $\left(\rho_{j}\right)_{j=0}^{\infty} \subset(0, \infty)$ such that

$$
\mu\left(\cup_{j=0}^{\infty} \rho_{j} S_{0}\right)<2 \mu\left(S_{0}\right) \quad \text { and } \quad \nu\left(\cup_{j=0}^{\infty} \frac{1}{\rho_{j}} \Sigma_{0}\right)<2 \nu\left(\Sigma_{0}\right) .
$$

Proof. Let $S_{1}$ be a measurable subset of $\mathbb{R}_{+}$of finite Lebesgue measure such that $S_{0} \subset S_{1}$. Define $h: \mathbb{R}_{+} \rightarrow \mathbb{R}_{+}$by $h(\rho)=\mu\left(S_{1} \cup \rho S_{0}\right)$. Since $\chi_{\rho S_{0}}$ and $\chi_{S_{1}}$ are in $L^{2}\left(\mathbb{R}_{+}, \mu\right)$, we may express $h$ in terms of scalar product in $L^{2}\left(\mathbb{R}_{+}, \mu\right)$

$$
h(\rho)=\left\|\chi_{\rho S_{0}}-\chi_{S_{1}}\right\|_{L^{2}\left(\mathbb{R}_{+}, \mu\right)}^{2}+\left\langle\chi_{\rho S_{0}}, \chi_{S_{1}}\right\rangle_{L^{2}\left(\mathbb{R}_{+}, \mu\right)} .
$$

The function $\rho \mapsto h(\rho)$ is a continuous function on $(0, \infty)$. We deduce that there exist an infinite sequence of distinct numbers $(\rho)_{j=0}^{\infty} \subset(0, \infty)$, with $\rho_{0}=1$ such that $\mu\left(\cup_{j=0}^{\infty} \rho_{j} S_{0}\right)<2 \mu\left(S_{0}\right)$. We can follow the same techniques to prove that

$$
\nu\left(\bigcup_{j=0}^{\infty} \frac{1}{\rho_{j}} \Sigma_{0}\right)<2 \nu\left(\Sigma_{0}\right) .
$$

We are now in position to prove Benedicks-type theorem for the Sturm-Liouville transform.

Theorem 3.1. Let $S$ and $\Sigma$ be a pair of measurable subsets of $\mathbb{R}_{+}$, with $0<\mu(S)$, $\nu(\Sigma)<\infty$, then the pair $(S, \Sigma)$ is weakly annihilating pair.

Proof. Suppose that there exist $f_{0} \neq 0$ such that $S_{0}=\operatorname{supp} f_{0}$ and $\Sigma_{0}=\operatorname{supp} \mathcal{F}\left(f_{0}\right)$ have both finite measure $0<\mu\left(\operatorname{supp} f_{0}\right), \nu\left(\operatorname{supp} \mathcal{F}\left(f_{0}\right)\right)<\infty$. From Lemma 3.2 we can find an infinite sequence of distinct numbers $(\rho)_{j=0}^{\infty} \subset(0, \infty)$, with $\rho_{0}=1$, such that, if we denote by $S=\cup_{j=0}^{\infty} \rho_{j} \operatorname{supp} f_{0}$ and $\Sigma=\cup_{j=0}^{\infty} \frac{1}{\rho_{j}} \operatorname{supp} \mathcal{F}\left(f_{0}\right)$ we have $\mu(S)<2 \mu\left(S_{0}\right)$, $\nu(\Sigma)<2 \nu\left(\Sigma_{0}\right)$.

Put $f_{i}=D_{\rho_{i}} f_{0}$, so that $\operatorname{supp} f_{i}=\rho_{i} \operatorname{supp} f_{0}$. As $\mathcal{F}\left(f_{i}\right)=D_{\frac{1}{\rho_{i}}} \mathcal{F}\left(f_{0}\right)$ we have $\operatorname{supp} \mathcal{F}\left(f_{i}\right)=\frac{1}{\rho_{i}} \operatorname{supp} \mathcal{F}\left(f_{0}\right)$. Since $\operatorname{supp} \mathcal{F}\left(f_{0}\right)$ has finite measure, $f_{0} \in \mathcal{C}_{0}\left(\mathbb{R}_{+}\right)$. It follows from Lemma 3.1 that $\left(f_{i}\right)_{i=0}^{\infty}$ are linearly independent vectors belonging to $\operatorname{Im} P_{S} \cap \operatorname{Im} Q_{\Sigma}$ which contradicts $(2.6)$. Then, $(S, \Sigma)$ is weakly annihilating.

Theorem 3.2 (Benedicks-type theorem). Let $S$ and $\Sigma$ be a pair of measurable subsets of $\mathbb{R}_{+}$with $0<\mu(S), \nu(\Sigma)<\infty$, then the pair $(S, \Sigma)$ is strong annihilating pair.

Proof. Assume there is no such constant $C(S, \Sigma)$. We can find a sequence $f_{n} \in$ $L^{2}\left(\mathbb{R}_{+}, \mu\right)$ of norm 1 weakly convergent in $L^{2}\left(\mathbb{R}_{+}, \mu\right)$ with some limit $f$ such that

$$
\operatorname{supp} f_{n} \subset S \text { and }\left\|\chi_{\Sigma^{c}} \mathcal{F}\left(f_{n}\right)\right\|_{L^{2}\left(\mathbb{R}_{+}, \nu\right)} \rightarrow 0 \text { as } n \rightarrow \infty .
$$

Since $\mathcal{F}\left(f_{n}\right)$ is the scalar product of $f_{n}$ and $\chi_{S} \varphi_{\lambda}(\cdot)$, it follows that $\mathcal{F}\left(f_{n}\right)$ converge to $\mathcal{F}(f)$. Since $\left|\mathcal{F}\left(f_{n}\right)\right|$ as bounded by $\sqrt{\mu(S)}$, it follows from Lebesgue's theorem that $\mathcal{F}\left(f_{n}\right) \chi_{\Sigma}$ converges to $\mathcal{F}(f)$ in $L^{2}\left(\mathbb{R}_{+}, \nu\right)$ and the limit $f$ has norm 1 . But the function $f$ has support in $S$ and spectrum in $\Sigma$, since $(S, \Sigma)$ is a weak annihilating pair, it follows that $f=0$, which gives a contradiction. 
Acknowledgements. The authors are deeply indebted to the reviewers for providing constructive comments and helps in improving the contents of this article.

\section{REFERENCES}

[1] A. Achak, R. Daher and H. Lahlali, Beurling's theorem for Bessel-Struve transform, C. R. Math. Acad. Sci. Paris 354(1) (2016), 81-85.

[2] M. Benedicks, On Fourier transforms of function supported on sets of finite Lebesgue measure, J. Math. Anal. Appl. 106 (1985), 180-183.

[3] A. Beurling, The Collect Works of Arne Beurling, Birkhäuser, Boston, 1989.

[4] A. Bonami and B. Demange, A survey on uncertainty principles related to quadratic forms, Collect. Math. 2 (2006), 1-36.

[5] M. G. Cowling and J. F. Price, Generalizations of Heisenberg Inequality, Lecture Notes in Mathematics 992 Springer, Berlin, 1983, 443-449.

[6] D. L. Donoho and P. B. Stark, Uncertainty principles and signal recovery, SIAM J. Appl. Math. 49 (1989), 906-931.

[7] G. B. Folland and A. Sitaram, The uncertainty principle a mathematical survey, J. Fourier Anal. Appl. 3 (1997), 207-238.

[8] S. Ghobber and Ph. Jaming, Uncertainty principles for integral operators, Studia Math. 220 (2014), 197-220.

[9] G. H. Hardy, A theorem concerning Fourier transform, J. London Math. Soc. 8 (1933), 227-231.

[10] V. Havin and B. Jöricke, The Uncertainty Principle in Harmonic Analysis, Springer-Verlag, Berlin, 1994.

[11] T. Kawazoe and H. Mejjaoli, Uncertainty principles for the Dunkl transform, Hiroshima Math. J. 40 (2010), 241-268.

[12] H. J. Landau and W. L. Miranker, The recovery of distored band-limited signal, J. Math. Anal. App. 2 (1961), 97-104.

[13] H. J. Landau and H. O. Pollak, Prolate spheroidal wave functions, Fourier analysis and uncertainty II, Bell System Technical Journal 40 (1961), 65-84.

[14] H. Mejjaoli Generalized Dunkl-Sobolev spaces of exponential type and applications, Journal of Inequalities in Pure and Applied Mathematics 10(2) (2009), 24 pages.

[15] A. Miyachi, A generalization of theorem of Hardy, Harmonic Analysis Seminar, Izunagaoka, Shizuoka-Ken, Japan, 1997, 44-51.

[16] N. Ben Salem. and A. Rashed Nasr, Heisenberg-type inequalities for the Weinstein operator, Integral Transforms Spec. Funct. 26(9) 2015, 700-718.

[17] A. Papoulis, A new algorithm in spectral analysis and band-limited extrapolation, IEEE Trans. Circuits Syst. I. Regul. Pap. 9 (1975), 735-742.

[18] D. Slepian and H. O. Pollak, Prolate spheroidal wave functions, Fourier analysis and uncertainty I, Bell System Technical Journal 40 (1961), 43-63.

[19] F. Soltani, $L^{p}$ uncertainty principles on Sturm-Liouville Hypergroups, Acta Math. Hungar. 142(2) (2014), 433-443.

[20] E. M. Stein, Interpolation of linear operators, Trans. Amer. Math. Soc. 83 (1956), 482-492.

[21] K. Tosio, Perturbation Theory for Linear Operators, Springer, New York, 1966. 
${ }^{1}$ Department of Mathematics, FACUlTy OF SCIENCES AÏN CHOCK,

University of Hassan II Casablanca 20100, Morocco

Email address: a.abouelaz@fsac.ac.ma

Email address: achakachak@hotmail.fr

Email address: r.daher@fsac.ac.ma

Email address: safouanenajat@live.fr 